\title{
Reconfiguration of Radial Electric Power Distribution System via a Scatter Search Algorithm
}

\author{
D. Rupolo and J. R. S. Mantovani
}

\begin{abstract}
In this paper, to solve the reconfiguration problem of radial distribution systems a scatter search, which is a metaheuristic-based algorithm, is proposed. In the codification process of this algorithm a structure called node-depth representation is used. It then, via the operators and from the electrical power system point of view, results finding only radial topologies. In order to show the effectiveness, usefulness, and the efficiency of the proposed method, a commonly used test system, 135-bus, and a practical system, a part of Sao Paulo state's distribution network, 7052 bus, are conducted. Results confirm the efficiency of the proposed algorithm that can find high quality solutions satisfying all the physical and operational constraints of the problem.
\end{abstract}

Keywords- Scatter Search Algorithm, Distribution Power System Reconfiguration, Exponential Load Model, Node-depth Representation.

\section{INTRODUÇÃO}

$\mathrm{O}_{\mathrm{r}}^{\mathrm{s}}$ S SISTEMAS aéreos de distribuição de energia elétrica possuem estruturas malhadas, mas operam de forma radial. Sua operação radial é realizada através de chaves de manobras, localizadas em pontos estratégicos da rede que operam no estado normalmente aberto (NA) e normalmente fechado (NF). A operação dos sistemas de distribuição de forma radial tem como objetivo facilitar fatores relacionados com a coordenação dos dispositivos de proteção, como também reduzir as correntes de curto-circuito [1].

Os sistemas de distribuição de energia elétrica podem ser reconfigurados através das chaves de manobras, com a finalidade de encontrar a topologia que opere no estado de mínimas perdas [2]. A reconfiguração consiste na alteração da topologia da rede através da mudança do estado das chaves NA e NF, com a condição de manter-se a radialidade do sistema e o fornecimento de energia a todos os pontos de carga. A reconfiguração do sistema de distribuição é uma ação de planejamento de curto prazo que permite melhorar as condições de operação da rede em função do crescimento da carga, possibilitando melhorar os níveis de tensão nos pontos de carga, propiciar uma maior confiabilidade ao sistema, como também permite realizar o balanceamento de cargas entre os alimentadores em caso de contingências [3].

O problema de reconfiguração de sistemas de distribuição de energia elétrica radiais é um problema de programação não linear inteiro misto [4]. Sendo de natureza combinatória, a busca por soluções factíveis e de qualidade é extremamente

D. Rupolo, Universidade Estadual Paulista Júlio de Mesquita Filho (UNESP), Ilha Solteira, São Paulo, Brasil, rupolo.diogo@gmail.com

J. R. S. Mantovani, Universidade Estadual Paulista Júlio de Mesquita Filho (UNESP), Ilha Solteira, São Paulo, Brasil, mant@dee.feis.unesp.br complexa, devido à alta quantidade de soluções presentes no espaço de busca, das quais, muitas destas não satisfazem as restrições físicas e operacionais do sistema, tais como radialidade, limites máximos e mínimos de magnitude de tensão nos pontos de carga, limites máximos de magnitude de corrente no circuito, entre outras [3].

$\mathrm{Na}$ busca de encontrar as topologias do sistema que operem no estado de mínimas perdas são propostas na literatura especializada várias técnicas, entre elas, algoritmos de otimização clássica, heurísticas, meta-heurística, entre outras. Inicialmente Merlin e Back [2] utilizaram duas propostas, sendo uma heurística construtiva e um algoritmo de otimização clássica com o objetivo de encontrar topologias que reduzissem as perdas no sistema de distribuição. Civanlar et. al. [5] propuseram a utilização de técnicas heurísticas para alterar a topologia operacional da rede e encontrar soluções que minimizassem as perdas de potência ativa. Baran e Wu [3] modelaram o problema de reconfiguração de redes para reduzir as perdas do sistema como um problema de programação inteira. Shirmohammad [4] utilizou um algoritmo heurístico juntamente com um fluxo de potência capaz de encontrar as soluções com as menores perdas no sistema. Dentre os principais trabalhos que utilizam metaheurísticas para encontrar as topologias que operassem no estado mínimo de perdas dos sistemas de distribuição na literatura destacam-se: Nara e Kitagawa [6] que utilizaram a meta-heurística Simulated Annealing, Nara et. al. [7] utilizaram o Algoritmo Genético e ZHANG [8] utilizou a meta-heurística Tabu Search.

Neste artigo, com o objetivo de encontrar soluções de qualidade para o problema de reconfiguração de redes de distribuição radiais de energia elétrica, que reduzam as perdas do sistema e melhorem as condições operacionais da rede, propõe-se o uso da meta-heurística busca dispersa (BD). A meta-heurística busca dispersa é um método de busca evolucionário que busca soluções para problemas de otimização através de critérios de intensificação e diversificação. Nas últimas décadas esta meta-heurística vem sendo largamente utilizada e provando ser eficaz em uma variedade de problemas de otimização [9]. Contudo, a aplicação da mesma na solução de problemas de planejamento de sistemas elétricos ainda é pouco difundida. A restrição de radialidade, considerando que o problema de otimização é resolvido através de meta-heurística, é considerada implicitamente através da representação nó profundidade, que é o sistema de codificação do conjunto de soluções [10]. Esta codificação possui operadores que permitem a geração de topologias sempre radiais e conexas para cada alimentador do sistema de distribuição, quando aplicada a outra topologia radial do sistema elétrico [10]. Para comprovar a eficiência da técnica utilizada, apresentam-se os resultados para um sistema 
de teste de 135 barras e um sistema real de 7052 barras.

O presente artigo está estruturado da seguinte forma: a seção I é introdutória, na qual se apresenta uma breve introdução do problema de reconfiguração e os principais trabalhos da literatura na área, com vistas a encontrar as topologias com o mínimo estado de perdas para os sistemas de distribuição. $\mathrm{Na}$ seção II apresenta-se o modelo matemático do problema de reconfiguração, juntamente com o modelo exponencial de cargas. Na seção III apresenta-se o tipo de codificação utilizada para os sistemas de distribuição de energia elétrica radiais. $\mathrm{Na}$ seção IV apresenta-se o algoritmo de reconfiguração proposto, composto das cinco etapas fundamentais da meta-heurística busca dispersa. Na seção $\mathrm{V}$ apresentam-se os resultados encontrados para os sistemas testados e na seção VI apresentam-se as conclusões deste trabalho.

\section{Modelo Matemático do Problema de RECONFIGURAÇÃo DE SISTEMAS DE DisTRIBUIÇÃO DE ENERGIA ELÉTRICA RADIAIS}

O modelo matemático do problema de reconfiguração de sistemas de distribuição de energia elétrica radiais pode ser formulado genericamente como:

$$
\left.\operatorname{Min} C_{k}=\sum_{p=1}^{P}\left(\sum_{i j=1}^{N_{C}}\left(R_{i j} \cdot I_{i j}{ }^{2}\right)\right) \cdot T_{p} \cdot C_{p}\right)
$$

sujeito às seguintes restrições:

$$
\begin{array}{ll}
P_{S_{i}}-P_{d_{i}}-\sum_{\left(j \in \Omega_{b i}\right)}\left(x_{i j} P_{i j}\right)=0 & \forall i \in \Omega_{b} \\
Q_{S_{i}}-Q_{d_{i}}-\sum_{\left(j \in \Omega_{b i}\right)}\left(x_{i j} Q_{i j}\right)=0 & \forall i \in \Omega_{b} \\
V_{\min } \leq V_{i} \leq V_{\max } & \forall i \in \Omega_{b} \\
I_{i j} \leq I_{\max } & \forall i j \in \Omega_{l} \\
x_{i j} \in\{0,1\} & \forall i j \in \Omega_{l} \\
\sum_{(i j) \in \Omega_{l}} x_{i j}=n_{s}-1 & \\
P_{d i}=P_{d_{0 i}} \cdot\left|\overline{V_{l}}\right|^{\alpha} & \\
Q_{d i}=Q_{d_{0 i}} \cdot\left|\overline{V_{l}}\right|^{\beta} &
\end{array}
$$

em que:

$C_{k}$ : custo das perdas de energia para uma determinada configuração $k$ do sistema.

$R_{i j}$ : resistência no circuito entre as barras $i$ e $j$;

$I_{i j}$ : magnitude de corrente no circuito entre as barras $i$ e $j$;

$I_{\max }$ : magnitude de corrente máxima no circuito entre as barras $i$ e $j$;
$N_{C}$ : número de circuitos da configuração $k$;

$P_{S_{i}}$ : potência ativa fornecida pela subestação na barra $i$;

$P_{d_{i}}$ : demanda de potência ativa na barra $i$;

$Q_{S_{i}}$ : potência reativa fornecida pela subestação da barra $i$;

$Q_{d_{i}}$ : demanda de potência reativa na barra $i$;

$P_{i j}$ : fluxo de potência ativa que sai da barra $i$ para a barra

$P_{d_{0 i}}$ : potência ativa no valor da tensão nominal;

$Q_{i j}$ : fluxo de potência reativa que sai da barra $i$ para barra

$\dot{Q}_{d_{0 i}}$ : potência reativa no valor da tensão nominal;

$x_{i j}$ : variável binária que determina se o circuito entre as

barras $i$ e $j$ está aberto ou fechado;

$\Omega_{b}$ : conjunto de barras do sistema;

$\Omega_{b i}$ : conjunto de barras conectadas na barra $i$;

$\Omega_{l}$ : conjunto de circuitos;

$n_{s}$ : número de setores do sistema

$V_{\text {min }}$ : magnitude de tensão mínima na barra $i$;

$V_{\text {max }}$ : magnitude tensão máxima na barra $i$;

$V_{i}$ : magnitude de tensão na barra $i$;

$\bar{V}_{l}$ : magnitude tensão na barra $i$ dividido pela tensão nominal do sistema;

$T_{p}$ : número de horas do período $p$;

$C_{p}$ : custo do $\mathrm{kW} / \mathrm{h}$ no período $p$;

$p$ : período de carga (leve, nominal ou pesado);

$P$ : número de períodos de carga;

A equação (1) representa a função objetivo do problema de reconfiguração, que tem por finalidade minimizar os custos relacionados com as perdas elétricas de energia de uma determinada topologia do sistema de distribuição radial, em um período anual, considerando os carregamentos leve, nominal e pesado. As equações (2) e (3) representam o balanço das potências ativa e reativa segundo as leis de Kirchoff. A equação (4) representa os limites máximo e mínimo de magnitude de tensão nos pontos de carga do sistema. A equação (5) representa os limites máximo de magnitude de corrente nos circuitos do sistema de distribuição. A equação (6) representa a variável de decisão binária $x_{i j}$, que assume os valores 0 e $1 \mathrm{e}$ representam os estados NA e NF das chaves de manobras. A equação (7) juntamente com as equações (2) e (3) são necessárias para garantir a restrição de radialidade do sistema. As equações (8) e (9) representam o modelo de cargas exponencial utilizado, em que a potência demandada varia de acordo com a tensão.

O modelo de cargas com potência constante é extensivamente utilizado na maioria dos trabalhos que trata do problema de reconfiguração, porém o modelo exponencial de cargas permite modelar de forma mais realista o sistema de distribuição em comparação com o modelo de cargas com 
potência constante, haja vista que os equipamentos ligados ao sistema podem operar com potência constante, corrente constante ou impedância constante [11].

Os índices $\alpha$ e $\beta$ apresentados na tabela I possibilitam a modelagem dos três tipos de cargas utilizados pelos equipamentos ligados ao sistema, pois se $\alpha=0$ e $\beta=0$, tem-se o modelo de carga com potência constante, se $\alpha=1$ e $\beta=1$ tem-se o modelo de cargas com corrente constante e se $\alpha=2$ e $\beta=2$ temse o modelo de cargas com impedância constante. Portanto os valores dos índices apresentados na tabela I têm por objetivo incluir os três modelos de cargas utilizados pelos equipamentos do sistema de distribuição. A segmentação dos tipos de consumidores em industriais, comerciais e industriais busca associar os índices $\alpha$ e $\beta$ com o respectivo modelo de cargas segundo os equipamentos presentes em cada segmento de consumo [12].

TABELA I. MODELO DE CARGA E SEUS VALORES EXPONENCIAIS.

\begin{tabular}{c|c|c}
\hline Modelo de carga & $\boldsymbol{\alpha}$ & $\boldsymbol{\beta}$ \\
\hline Industrial & 0,18 & 6 \\
\hline Comercial & 1,04 & 4,19 \\
\hline Residencial & 1,50 & 3,15 \\
\hline
\end{tabular}

\section{CODIFICAÇÃo DO PROBLEMA}

A codificação é uma importante ferramenta para qualquer problema que envolva meta-heurísticas. Utiliza-se neste trabalho como sistema de codificação a representação nó profundidade [10]. Esta codificação possibilita o uso dos operadores PAO (do inglês, Preserve Ancestor Operator), CAO (do inglês, Change Ancestor Operator) e EHR [13] (do inglês, Evolutionary History Recombination) para a geração de soluções radiais quando aplicadas aos sistemas de distribuição de energia elétrica.

Os operadores PAO e CAO utilizam critérios baseados em teoria dos grafos para podar e transferir seções entre os alimentadores dos sistemas de distribuição. Estes operadores quando aplicados a uma topologia radial do sistema, geram outra topologia sempre radial e conexa, sendo que as topologias geradas pelo operador PAO são mais similares com a topologia da qual são geradas, enquanto que topologias geradas com o operador CAO são bem mais diversas em relação à topologia inicial [10]. O operador EHR permite a combinação de duas soluções radiais, gerando uma terceira solução radial e conexa [13].

\section{Algoritmo de Busca Dispersa DedicAdo aO PROBLEMA DE RECONFIGURAÇÃO}

O algoritmo de busca dispersa dedicado ao problema de reconfiguração de sistemas de distribuição de energia elétrica radiais consiste em cinco etapas fundamentais:

Geração de soluções com diversidade: Nesta etapa aplicase o operador $\mathrm{CAO}$ na topologia inicial do sistema de distribuição radial gerando um conjunto inicial $P$ de dimensão $P_{\text {Size }}$. As soluções deste conjunto devem possuir um determinado grau de diversidade. Neste sentido a diversidade entre as soluções geradas é imposta ao utilizarem-se os operadores da representação nó profundidade. Ao se transferir os setores do sistema de distribuição entre os alimentadores consideram-se relações de probabilidade, de forma que todos tenham a mesma probabilidade de serem escolhidos para serem podados. No início do processo todos os alimentadores têm a mesma chance de serem escolhidos e nas iterações seguintes esta probabilidade é inversamente proporcional aos alimentadores que se encontram selecionados.

Melhoria local: $\mathrm{Na}$ etapa de melhoria local aplica-se o operador PAO em uma solução base com a finalidade de encontrar na vizinhança desta topologia uma solução que possa melhorar o valor da função objetivo. Este operador gera uma solução bem semelhante à solução à qual é aplicada e, portanto podem-se gerar vários vizinhos com este método.

Construção e atualização do conjunto de referência: No conjunto de referência, também denominado de RefSet, escolhem-se as $b_{1}$ melhores soluções de qualidades considerando o valor da função objetivo. Para encontrar as soluções que minimizam as perdas de energia é utilizado o fluxo de potência tipo varredura direta e reversa de Shirmohammad [14]. As $b_{2}$ soluções são escolhidas através de um critério de diversidade. Esta diversidade é imposta considerando a quantidade de barras diferentes em relação ao mesmo alimentador ao compararem-se duas topologias do sistema. A atualização do conjunto de referência é realizada após as etapas de geração de subconjuntos, combinação e melhoria local, onde são selecionadas as melhores soluções encontradas.

Geração de subconjuntos: Nesta etapa geram-se todos os subconjuntos com duas soluções que compõem o conjunto de referência. Estas soluções são agrupadas no conjunto NovosSubconjuntos. Portanto nesta etapa, apenas são agrupadas duas soluções para posteriormente serem submetidas à etapa de combinação.

Combinação: Nesta etapa utiliza-se o operador EHR para realizar uma combinação convexa entre as soluções dos subconjuntos formados na etapa anterior. Através de duas soluções radiais e conexas, gera-se uma terceira solução com as mesmas propriedades [13]. Após a etapa de combinação e melhoria local, todas as soluções são armazenadas no conjunto Pool.

O diagrama de blocos do algoritmo de busca dispersa para a reconfiguração de redes de distribuição de energia elétrica é apresentado na Figura 1. 


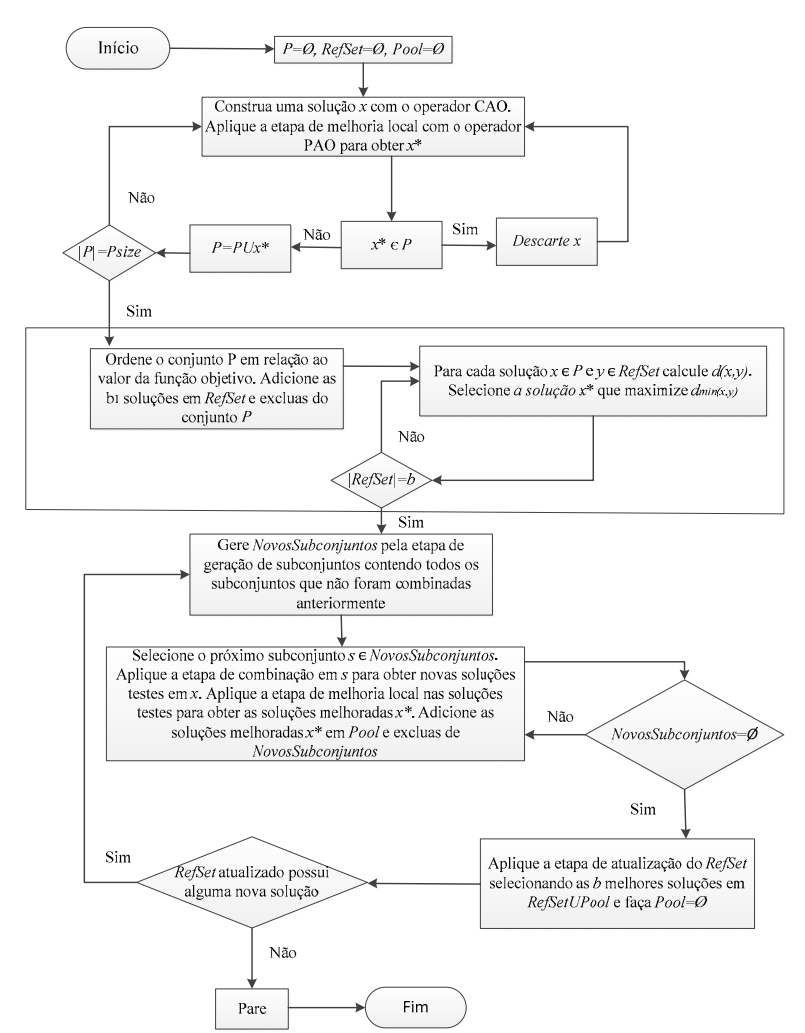

Figura 1. Diagrama de blocos do algoritmo de BD proposto para o problema de reconfiguração.

\section{Resultados}

O algoritmo de busca dispersa dedicado ao problema de reconfiguração de sistemas de distribuição é implementado em linguagem de programação $\mathrm{C}++$ utilizando um micro computador com processador Core i7 de $2,53 \mathrm{GHz}$ e $4 \mathrm{~GB}$ de memória RAM. Para testar a metodologia proposta e verificar a eficiência computacional do algoritmo, são realizadas simulações em um sistema teste de distribuição de 135 barras e um sistema real de 7052 barras, ambos pertencentes à rede urbana do sistema de distribuição.

Na função objetivo considera-se um período anual de 8760 horas dividido em três tipos de carregamento, sendo: 2000 horas para o carregamento leve, considerando $50 \%$ da potência instalada do sistema, 5760 horas para o carregamento nominal, considerando $70 \%$ da potência instalada do sistema e 1000 horas para o carregamento pesado, considerando $100 \%$ da potência instalada do sistema. No modelo exponencial de cargas, consideram-se $70 \%$ das cargas como residenciais, $20 \%$ das cargas como comerciais e $10 \%$ das cargas como industriais. Para os custos das perdas são utilizados os seguintes valores do kWh: US\$ 0,16 para o carregamento leve, US\$ 0,20 para o carregamento médio e US\$ 0,25 para o carregamento pesado.

\section{A - Sistema teste de 135 barras}

Este sistema possui 135 barras, 2 subestações, 8 alimentadores, potência nominal de $100 \mathrm{MVA}$, tensão nominal de $13,8 \mathrm{kV}, 135$ chaves normalmente fechadas, 21 chaves normalmente abertas, com uma demanda de potência ativa de
$18.313,809 \mathrm{~kW}$ e reativa de $9.384,827 \mathrm{kVAr}$ [15]. Neste sistema considera-se que existem chaves de manobras entre todas as barras para verificar a eficiência computacional do algoritmo em relação à complexidade do problema. Sua topologia inicial é mostrada na Figura 2, em que as linhas contínuas representam os circuitos que estão fechados no sistema e as linhas tracejadas representam os circuitos que estão abertos, através das chaves de manobras.

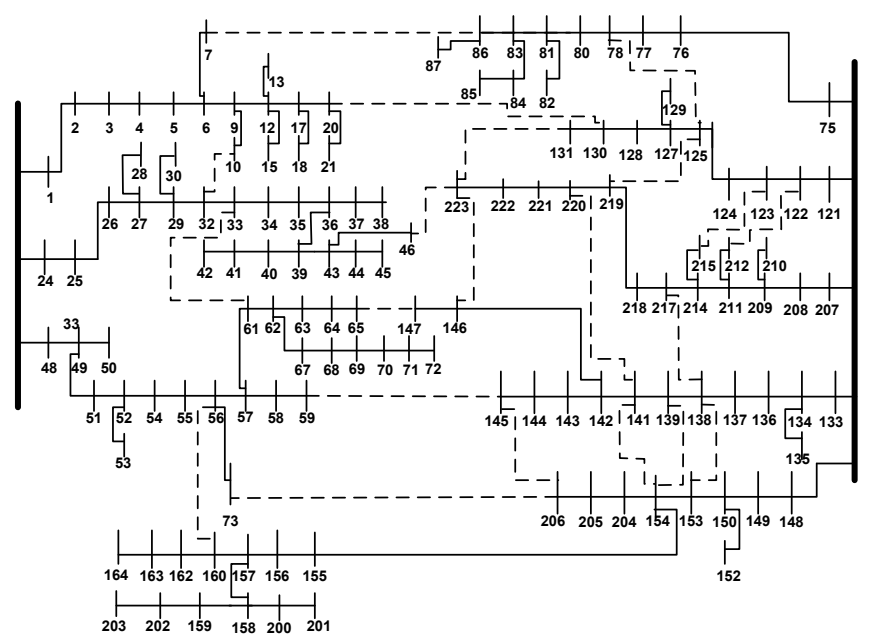

Figura 2. Topologia inicial do sistema de 135 barras.

Em relação aos parâmetros dos conjuntos da meta-heurística busca dispersa, para o sistema teste de 135 barras são utilizadas 50 soluções no conjunto inicial $P$ e no conjunto de referência utilizam-se 10 soluções, sendo que destas, 5 soluções são selecionadas pelo critério de qualidade e 5 soluções pelo critério de diversidade.

$\mathrm{Na}$ tabela II apresentam-se os custos relacionados à configuração inicial $(\mathrm{CI})$ e a melhor solução encontrada pelo algoritmo após a reconfiguração (CR), utilizando o modelo exponencial de cargas.

TABELA II. RESULTADOS DA RECONFIGURAÇÃO DO SISTEMA DE 135 BARRAS.

\begin{tabular}{|c|c|c|}
\hline Conf. & Circuitos abertos & $\begin{array}{c}\text { Custos } \\
\text { (US\$) }\end{array}$ \\
\hline \multirow{6}{*}{ CI } & $7-86 ; 10-32 ; 20-130 ; 33-61 ; 46-$ & \\
& $223 ; 56-160 ; 59-145 ; 65-146 ; 73-$ & \\
& $206 ; 78-125 ; 122-212 ; 123-215 ;$ & \\
& $125-219 ; 131-223 ; 138-153 ; 139-$ & $259.343,50$ \\
& $154 ; 141-154 ; 141-220 ; 145-206 ;$ & \\
& $147-223 ; 217-138 ;$ & \\
\hline \multirow{6}{*}{ CR } & $6-7 ; 10-32 ; 20-130 ; 43-46 ; 57-61 ;$ & \\
& $59-145 ; 78-125 ; 125-219 ; 131-223 ;$ & \\
& $137-138 ; 138-139 ; 138-217 ; 139-$ & $232.355,38$ \\
& $154 ; 141-154 ; 141-220 ; 144-145 ;$ & \\
& $147-223 ; 154-204 ; 155-156 ; 211-$ & \\
& $212 ; 214-215 ;$ & \\
\hline
\end{tabular}

Na Figura 3 apresenta-se a topologia encontrada após o processo de reconfiguração, em que as linhas contínuas representam os circuitos que estão fechados no sistema e as linhas tracejadas representam os circuitos que estão abertos, através das chaves de manobras. 


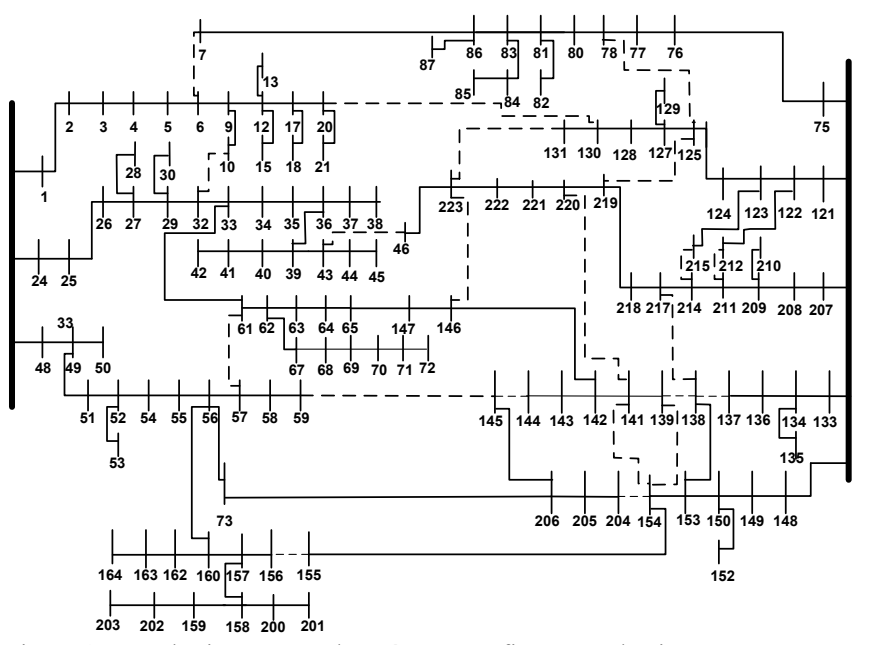

Figura 3. Topologia encontrada após a reconfiguração do sistema.

O tempo computacional do algoritmo é de 16,512 segundos. Verifica-se que há uma redução de $10,4 \%$ nos custos de operação do sistema quando se compara a topologia inicial com a topologia encontrada após o processo de reconfiguração. $\mathrm{O}$ algoritmo realiza 16 iterações para encontrar a solução apresentada.

O segundo teste considera um aumento desordenado de $20 \%$ nas demandas ativa e reativa em $30 \%$ das barras do sistema de distribuição. Desta forma é preciso reconfigurar novamente o sistema e encontrar a topologia que apresente o estado de mínimas perdas, haja vista que não se pode garantir que a topologia com perdas mínimas seja a mesma encontrada anteriormente. $\mathrm{Na}$ tabela III apresentam-se os resultados encontrados.

TABELA III. RESULTADOS: RECONFIGURAÇÃO COM AUMENTO NAS DEMANDAS DE CARGAS.

\begin{tabular}{|c|c|c|}
\hline Conf. & Chaves abertas & $\begin{array}{c}\text { Custos } \\
\text { (US\$) }\end{array}$ \\
\hline \multirow{6}{*}{ CI } & $6-7 ; 10-32 ; 20-130 ; 43-46 ; 57-61 ;$ & \\
& $59-145 ; 78-125 ; 125-219 ; 131-223 ;$ & \\
& $137-138 ; 138-139 ; 138-217 ; 139-$ & $267.419,50$ \\
& $154 ; 141-154 ; 141-220 ; 144-145 ;$ & \\
& $147-223 ; 154-204 ; 155-156 ; 211-$ & \\
\hline \multirow{5}{*}{ CR } & $212 ; 214-215 ;$ & \\
& $6-7 ; 10-32 ; 20-130 ; 46-223 ; 57-61 ;$ & \\
& $59-145 ; 65-146 ; 78-125 ; 125-219 ;$ & \\
& $137-138 ; 138-217 ; 139-154 ; 141-$ & $266.602,10$ \\
& $154 ; 141-220 ; 144-145 ; 147-223 ;$ & \\
& $154-204 ; 155-156 ; 211-212 ; 214-$ & \\
\hline
\end{tabular}

$\mathrm{Na}$ Figura 4 apresenta-se a topologia encontrada após $\mathrm{o}$ aumento de $20 \%$ da demanda de cargas em parte do sistema. Desta forma, verifica-se que é encontrada uma nova topologia ótima para o sistema que minimiza seus custos anuais relativos às perdas.

Um terceiro teste considera a presença de um gerador distribuído na barra 203 deste sistema, sendo de $1.000 \mathrm{~kW}$ de potência ativa e $600 \mathrm{kVAr}$ de potência reativa.

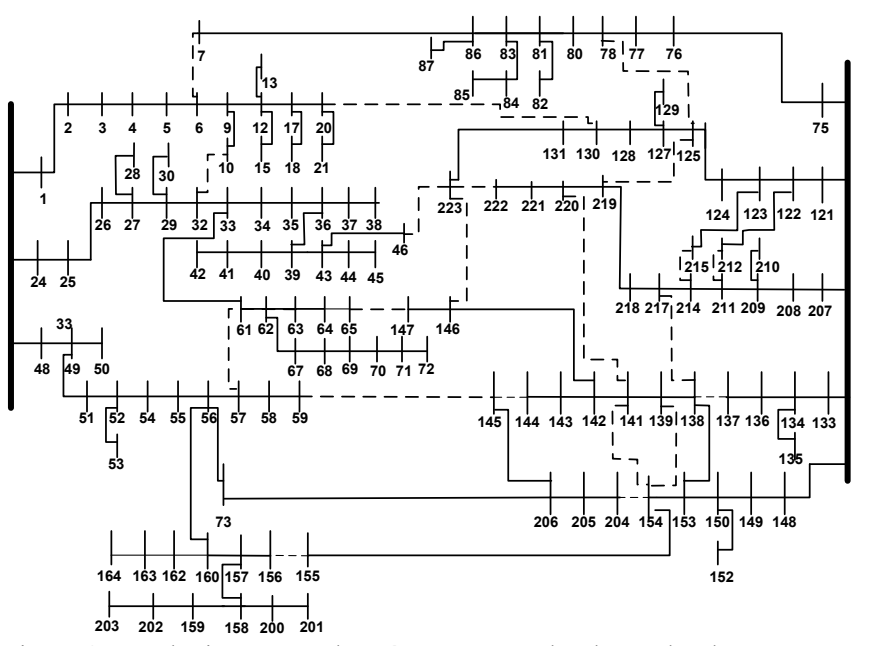

Figura 4. Topologia encontrada após o aumento das demandas de cargas.

Na tabela IV apresentam-se os custos relacionados com as perdas do sistema, utilizando o modelo exponencial de cargas, juntamente com a geração distribuída.

TABELA IV. RESULTADOS DO MODELO EXPONENCIAL DE CARGAS COM GERAÇÃO DISTRIBUÍDA.

\begin{tabular}{|c|c|c|}
\hline Conf. & Chaves abertas & $\begin{array}{c}\text { Custos } \\
\text { (US\$) }\end{array}$ \\
\hline \multirow{6}{*}{ CI } & $7-86 ; 10-32 ; 20-130 ; 33-61 ; 46-$ & \\
& $223 ; 56-160 ; 59-145 ; 65-146 ; 73-$ & \\
& $206 ; 78-125 ; 122-212 ; 123-215 ;$ & $232.355,38$ \\
& $125-219 ; 131-223 ; 138-153 ; 138-$ & \\
& $217 ; 139-154 ; 141-154 ; 141-220 ;$ & \\
& $145-206 ; 147-223 ;$ & \\
\hline \multirow{6}{*}{ CR } & $6-7 ; 10-32 ; 20-130 ; 33-61 ; 46-$ & \\
& $223 ; 59-145 ; 63-64 ; 78-125 ; 125-$ & \\
& $219 ; 137-138 ; 138-217 ; 139-154 ;$ & $211.584,40$ \\
& $144-145 ; 147-223 ; 154-204 ; 157-$ & \\
& $160 ; 141-154 ; 141-220 ; 211-212 ;$ & \\
& $214-215 ; 222-223 ;$ & \\
\hline
\end{tabular}

Na Figura 5 apresenta-se a topologia encontrada com a presença do gerador distribuído:

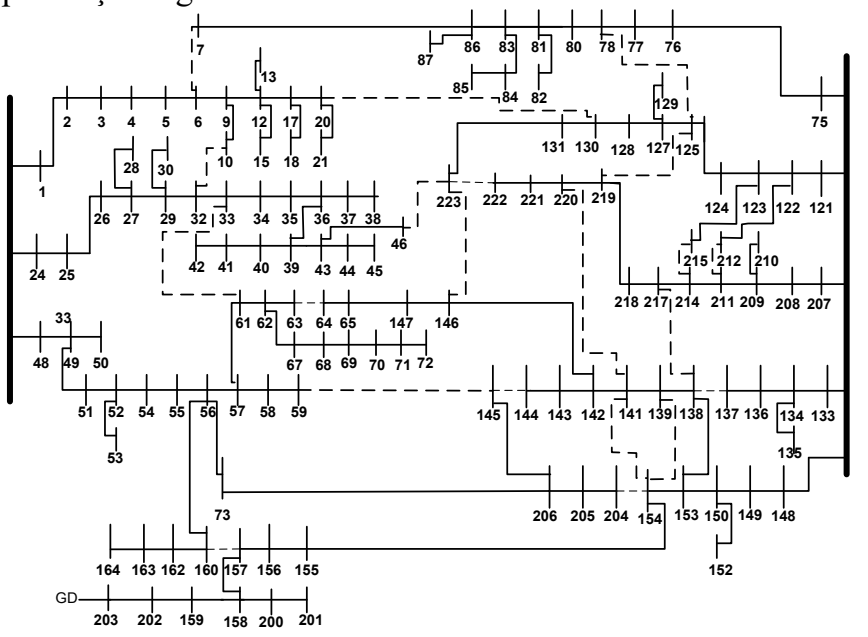

Figura 5. Topologia encontrada com a presença do gerador distribuído.

De acordo com a Figura $\mathbf{5}$, verifica-se que é encontrada uma nova topologia ótima para o sistema, com a presença do gerador 
distribuído. Os resultados mostram uma redução de $8,93 \%$ nos custos de operação do sistema, após a reconfiguração. Verificase também que há uma redução de $10,40 \%$ quando se conectam os geradores distribuídos no sistema, em relação à topologia base.

\section{B - Sistema Real de 7052 barras}

Este sistema possui 7052 barras, 9 alimentadores, potência nominal de $101570 \mathrm{kVA}, 708$ chaves de manobras, tensão base de $13,2 \mathrm{kV}$ e demanda total de $59726,76 \mathrm{~kW}$ de potência ativa e 29687,21 kVAr de potência reativa.

Os parâmetros utilizados para o algoritmo de busca dispersa são 100 soluções no conjunto inicial $P$ e 20 soluções no conjunto de referência, sendo 10 soluções de qualidade e 10 soluções de diversidade.

Os testes para este sistema consideram a reconfiguração do sistema em relação à topologia inicial, com aumento da demanda de cargas após um determinado período de tempo e com geração distribuída (GD). No aumento de cargas considera-se o aumento desordenado de $20 \%$ nas demandas de cargas em $20 \%$ nos pontos de demanda do sistema de distribuição. No sistema com geração distribuída considera-se a presença de quatro geradores distribuídos, sendo estes de $1.000 \mathrm{~kW}$ de potência ativa e $600 \mathrm{kVAr}$ de potência reativa. $\mathrm{Na}$ tabela $\mathrm{V}$ apresentam-se os resultados encontrados.

TABELA V. RESULTADOS DA RECONFIGURAÇÃO DO SISTEMA REAL DE 7052 BARRAS

\begin{tabular}{|c|c|c|c|}
\hline & $\begin{array}{c}\text { Sistema base } \\
\text { (US\$) }\end{array}$ & $\begin{array}{c}\text { Aumento das } \\
\text { demandas } \\
\text { (US\$) }\end{array}$ & GD (US\$) \\
\hline CI & $2.313 .174,50$ & $2.451 .196,00$ & $2.187 .890,00$ \\
\hline CR & $2.187 .787,50$ & $2.384 .325,50$ & $1.974 .101,50$ \\
\hline
\end{tabular}

De acordo com a tabela $\mathrm{V}$ verifica-se que houve uma redução de $5,42 \%$ nos custos de operação do sistema quando se compara a topologia inicial (CI) com a topologia encontrada após o processo de reconfiguração (CR). O tempo computacional do algoritmo é de aproximadamente 1792 segundos, com um total de 24 iterações. No aumento da demanda de cargas, verifica-se que é encontrada uma nova topologia ótima para o sistema que minimiza $2,72 \%$ seus custos anuais de perdas. No sistema com geração distribuída verifica-se que houve uma significativa redução de $9,77 \%$ dos custos anuais relativo às perdas de energia do sistema, após o processo de reconfiguração. Também verifica-se uma redução de $5,41 \%$ das perdas em relação a topologia inicial do sistema quando conectam-se os geradores distribuídos. Deve-se também ressaltar que as topologias encontradas com e sem geração distribuída após a reconfiguração dos sistemas de distribuição são diferentes uma da outra.

\section{CONCLUSÕES}

Neste trabalho propõe-se a implementação de um algoritmo de busca dispersa dedicado ao problema de reconfiguração de sistemas de distribuição de energia elétrica radiais, utilizando como codificação a representação nó profundidade, juntamente com o modelo exponencial de cargas. Os operadores da representação nó profundidade se mostraram adequados produzindo somente soluções radiais e conexas para cada alimentador do sistema de distribuição, juntamente com a metaheurística busca dispersa que permitiu através de seu método de busca encontrar soluções de excelente qualidade.

O modelo exponencial de cargas permitiu modelar o sistema de distribuição de forma mais realista encontrando-se assim resultados mais precisos e reais, tendo em vista que as cargas presentes no sistema operam com impedância constante, corrente constante e potência constante.

Os parâmetros de controle testados na dimensão dos conjuntos utilizados obtidos através de testes exaustivos mostraram-se eficientes, pois geraram soluções de boa qualidade para o problema.

$\mathrm{O}$ aumento de cargas sofrido no sistema após um período específico de tempo necessitou de uma nova configuração ótima, haja vista que a topologia em operação não apresentava mais o estado de mínimas perdas. Desta forma, nota-se que houve uma redução dos custos em comparação com a topologia que o sistema estava operando.

A geração distribuída permitiu reduzir as perdas do sistema, como também alterou a topologia para que o sistema operasse no estado de mínimas perdas.

Portanto, considera-se que a meta-heurística busca dispersa, juntamente com a codificação dada pela representação nó profundidade encontrou resultados de excelente qualidade para o problema de reconfiguração de sistemas de distribuição de energia elétrica radiais, atendendo todas as restrições físicas e operacionais do problema. Trata-se de uma técnica de solução eficiente e original para o problema de reconfiguração de redes.

\section{AgRAdecimentos}

Agradecimentos a CAPES e o CNPq (processo 305371/2012-6) pelo apoio financeiro.

\section{REFERÊNCIAS}

[1] T. GÖNEN, Electric Power Distribution System Engineering. New York, McGraw-Hill, 1986

[2] MERLIN, A.; BACK, H. Search for a minimal-loss operating spanning tree configuration in an urban power distribution system. Power System Computation Conference, Proceedings... Zurich: Cambridge, 1975. p. 118.

[3] BARAN, M. E.; WU, F. F. Network reconfiguration in distribution systems for loss reduction and load balancing. IEEE Transactions on Power Delivery, New York, v. 4, n.2, p. 1401-1407, 1989.

[4] SHIMOHAMMADI, D.; HONG, H. W. Reconfiguration of eletric distribution for resistive line loss reduction. IEEE Transactions on Power Delivery, New York, v. 4, n. 2, p. 1492-1498, 1989.

[5] CIVANLAR, S.; GRAINGER, J. J.; YIN, H.; LEE, S. S. H. Distribution feeder reconfiguration for loss reduction. IEEE Transactions on Power Delivery, New Orleans, v. 3, n. 3, p. 1217-1223, 1988.

[6] NARA, K.; KITAGAWA, M. Distribution systems loss minimum reconfiguration by simulated annealing method. Power System Control, Operation and Management, International Conference, Hon Kong, v. 2, 1991. p. 461-466

[7] NARA, K.; SHIOSE, A.; KITAGAWA, M.; ISHIRARA, T.; Implementation of genetic algorithm for distribution systems loss minimum reconfiguration. IEEE Transactions on Power Systems, New York, v. 7, n. 3, p. 1044-1051, 1992.

[8] ZHANG, D.; FU, Z.; ZHANG, L. An improved TS algorithm for lossminimum reconfiguration in large-scale distribution systems. Electric Power Systems Research, Lausanne, v. 77, n. 5-6, p. 685-694, 2007. 
[9] LAGUNA, M.; MARTÍ, R. Scater search: methodology and implementations in C. Boston: Kluwer Academic Publishers, 2003. p. 1291.

[10] DELBEM, A. C. B., CARVAlHO, A. de; POLICASTRO, C. A; PINTO, A. K. O.; HONDA, K.; GARCIA A. C. Node-depth encoding for evolutionary algorithms applied to network design. Genetic and Evolutionary Computation - GECCO, p. 678-687, 2004.

[11] RODEZNO, D. A. Q; Controle de Tensão e Reativos em Redes de Distribuição de Energia Elétrica. Dissertação de Mestrado. Universidade Estadual Paulista Julio de Mesquita Filho, 2014.

[12]IEEE Transactions on Power System, CASPER, S. G. (Ed.). Bibliography on load models for power flow and dynamic performance simulation. Piscataway, v. 10, n. 1, p.523-538, 1995.

[13] SANCHES, D. S.; LIMA, T. W.; SANTOS A. C.; DELBEM A. C. B., LONDON J. B. A. Jr. Node-Depth Encoding with Recombination for Multi-Objective Evolutionary Algorithm to Solve Loss Reduction Problem in Large-scale Distribution Systems. Power and Energy Society General Meeting, IEEE, 2012.

[14] SHIRMOHAMMAD, D. A.; HONG, H. W.; SEMLEY, A.; LUO, G. X. A Compensation-based power flow method for weakly meshed distribution and transmission networks, IEEE Transactions on Power Systems, Montreal, v. 3, n. 2, p. 753-762, 1988.

[15] MANTOVANI, J. R. S.; CASARI, F.; ROMERO R., Reconfiguração de sistema de distribuição radiais utilizando o critério de queda de tensão. SBA Controle e Automação, Porto Alegre, v. 11, n. 2, p. 150-159, 2000.

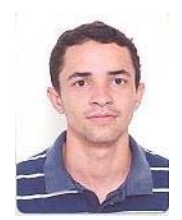

Diogo Rupolo é graduado em Matemática pela Universidade Estadual do Mato Grosso (UNEMAT), Sinop, Mato Grosso, Brasil, em 2011. Obteve o título de mestre em Engenharia Elétrica pela Universidade Estadual Paulista Julio de Mesquita Filho (UNESP), Ilha Solteira, São Paulo, Brasil, em 2013. Atualmente é aluno de doutorado em Engenharia Elétrica da Universidade Estadual Paulista Julio de Mesquita Filho (UNESP) e suas pesquisas se concentram na área de planejamento e otimização de redes de distribuição de energia elétrica.

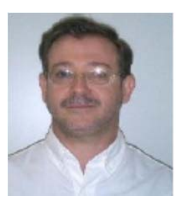

José Roberto Sanches Mantovani é graduado em Engenharia Elétrica pela Universidade Estadual Paulista (UNESP), Campus de Ilha Solteira, São Paulo, Brasil, em 1981. Pós-Graduado em Engenharia Elétrica em nível de mestrado pela Universidade Estadual de Campinas (UNICAMP), São Paulo, Brasil, 1987. Pós-Graduado, em nível de doutorado, em Engenharia Elétrica pela UNICAMP em 1995. Atualmente é professor titular do Departamento de Engenharia Elétrica (DEE) e membro do Laboratório de Planejamento de Sistemas de Energia Elétrica (LaPSEE) na UNESP. 